\title{
Judicialização do acesso aos serviços de saúde: análise de caso da Secretaria de Saúde de Pernambuco
}

\author{
Health's Judicialization: a case study of the Health Secretariat of Pernambuco \\ Judicialización de la Salud: análisis de caso de la Secretaría de Salud de Pernambuco
}

\author{
Fábio Henrique Cavalcanti de Oliveira ${ }^{1}$ \\ José Eudes de Lorena Sobrinho \\ Maria Cristina Sette de Lima $^{3}$ \\ Ulisses Ramos Montarroyos ${ }^{4}$ \\ Mirlene Giovanna Aragão Baía das Neves ${ }^{5}$ \\ Paula Rodrigues da Silva ${ }^{6}$ \\ Laís Navarro Xavier ${ }^{7}$ \\ Geraldo Jorge Filho ${ }^{8}$ \\ Diego Medeiros Guedes ${ }^{9}$ \\ Flavio José Cintra de Almeida ${ }^{10}$
}

Resumo. Objetivo: Este artigo objetivou analisar a judicialização do acesso aos serviços de saúde na Secretaria Estadual de Saúde de Pernambuco no ano de 2016. Métodos: Tratou-se de estudo transversal, quantitativo e analítico com dados do Núcleo de Ações Judiciais da Secretaria Estadual de Saúde de Pernambuco em 2016. As variáveis foram: o produto; o estabelecimento de atendimento; CID-10; municípios da comarca; município de residência; sexo; grupo do produto e condutor da ação. Resultados: Dos 2.560 processos, os medicamentos corresponderam a $63,5 \%$ das ações. $42 \%$ das demandas concentraram-

\footnotetext{
${ }^{1}$ Farmacêutico pela UFPE. Doutorando em Ciências da Saúde pela UPE. Mestre em Inovação Terapêutica pelo PPGIT/UFPE. Professor da Faculdade de Ciências Médicas, Universidade de Pernambuco. Recife, PE. Brasil. E-mail: fabiohcavalcanti@upe.br

${ }^{2}$ Cirurgião-dentista Doutor em Saúde Pública. Professor da Faculdade de Ciências Médicas, Universidade de Pernambuco. Recife, PE. Brasil. E-mail: eudes.lorena@upe.br

${ }^{3}$ Médica. Especialista em Planejamento e Gestão Organizacional. Professora da Faculdade de Ciências Médicas, Universidade de Pernambuco. Recife, PE. Brasil. E-mail: cristinasette@bol.com.br

${ }^{4}$ Graduação em Estatística, Mestre em Saúde pública, Doutor em Medicina Tropical, na UFPE. Professor da Faculdade de Ciências Médicas, Universidade de Pernambuco. Recife, PE. Brasil. E-mail: ulisses.montarroyos@upe.br

${ }^{5}$ Graduada em Saúde Coletiva. Residente em Saúde Coletiva, Faculdade de Ciências Médicas, Universidade de Pernambuco. Recife, PE. Brasil. E-mail: mirlene.neves@gmail.com

${ }^{6}$ Graduada em Saúde Coletiva. Residente em Atenção Básica, Centro Universitário Tabosa de Almeida, Caruaru, PE. Brasil. E-mail: paularodrigues2804@gmail.com

${ }^{7}$ Graduada em Saúde Coletiva. Pós-graduanda MBA Gestão Hospitalar e Gestão em Serviços de Saúde. Faculdade de Administração e Direito de Pernambuco, Universidade de Pernambuco. Recife, PE. Brasil. Email: laisnavarro_x@hotmail.com

8 Graduado em Administração. Especialista em contabilidade pública e controladoria governamental. Especialista em Direito Sanitário. Secretaria Estadual de Saúde de Pernambuco, Recife - PE. Brasil. E-mail: geraldo.filho@saude.pe.gov.br

${ }^{9}$ Farmacêutico. Mestrando em Inovação Terapêutica. Universidade Federal de Pernambuco, Recife - PE. Brasil. E-mail: diegomedeirosguedes@gmail.com

${ }^{10}$ Enfermeiro. Graduando em Odontologia. Centro Universitário Tabosa de Almeida, Caruaru, PE. Brasil. Email: fcintra45@gmail.com
} 
se na $1^{\text {a }}$ Região de Saúde; 39,3\% e 31,5\% foram provenientes de unidades públicas de saúde e filantrópicas, respectivamente. Os dados corroboram com achados da literatura quanto ao item mais demandado judicialmente e à tendência de concentração em regiões metropolitanas. Difere quanto à natureza do órgão demandante por ter sido neste caso o próprio poder público e as unidades de saúde pública. Conclusões: Incorporar os objetos das demandas judiciais na oferta regular dos serviços de saúde pode contribuir para a redução de processos e aumentar o acesso dos cidadãos aos direitos legais. Em alguns casos, garantir o cumprimento das normas já editadas favorece a tendência de diminuição da judicialização, sendo necessário para esta análise os estudos longitudinais.

Palavras-chave: Judicialização da Saúde. Economia da Saúde. Direito Sanitário.

Abstract. Objective: This article aimed to analyze the judicialization of access to health services in the State Department of Health of Pernambuco in the year 2016. Methodology: This was a cross-sectional, quantitative and analytical study with data from the Judicial Actions Center of the State Health Secretariat of Pernambuco in 2016. The variables were: the product; the establishment of care; ICD-10; municipalities of the region; municipality of residence; sex; product group and action driver. Results: Of the 2,560 cases, the medications corresponded to $63.5 \%$ of the actions. $42 \%$ of the demands were concentrated in the 1st Health Region; 39.3\% and 31.5\% came from public health and philanthropic units, respectively. The data corroborate with findings from the literature regarding the item most sued in court and the trend of concentration in metropolitan regions. It differs as to the nature of the complainant organ because in this case it was the public authority itself and the public health units. Conclusions: Incorporating the objects of lawsuits into the regular provision of health services can contribute to the reduction of processes and increase citizens' access to legal rights. In some cases, ensuring compliance with the standards already published favors the tendency to decrease the judicialization, being necessary for this analysis the longitudinal studies.

Keywords: Health's Judicialization. Health Economics. Health Law.

Resumén. Objetivo: Este artículo objetivó analizar la judicialización del acceso a los servicios de salud en la Secretaría Estatal de Salud de Pernambuco en el año 2016. Metodología: Se trató de estudio transversal, cuantitativo y analítico con datos del Núcleo de Acciones Judiciales de la Secretaría Estatal de Salud de Pernambuco en 2016. Las variables fueron: el producto; el establecimiento de atención; ICD-10; municipios de la comarca; municipio de residencia; el sexo; grupo del producto y conductor de la acción. Resultados: De los 2.560 procesos, los medicamentos correspondieron al 63,5\% de las acciones. El $42 \%$ de las demandas se concentró en la 1a Región de Salud; 39,3\% y 31,5\% provenían de unidades públicas de salud y filantrópicas, respectivamente. Discusión: Los datos corroboran con hallazgos de la literatura en cuanto al ítem más demandado judicialmente ya la tendencia de concentración en regiones metropolitanas. Difiere en cuanto a la naturaleza del órgano demandante por haber sido en este caso el propio poder público y las unidades de salud pública. Conclusiones: Incorporar los objetos de las demandas judiciales en la oferta regular de los servicios de salud puede contribuir a la reducción de procesos y aumentar el acceso de los ciudadanos a los derechos legales. En algunos casos, garantizar el cumplimiento de las normas ya editadas favorece la tendencia de disminución de la judicialización, siendo necesario para este análisis los estudios longitudinales. 
Palabras-Ilave: Judicialización de la Salud. Economía de la Salud. Derecho Sanitario.

\section{Introdução}

Progressos quanto à universalidade do direito à saúde foram elencados na Constituição Federal de 1988 (1), implicando na reorganização do sistema de saúde do país com regime jurídico diferenciado (2). Dispositivos legais publicados posteriormente, como a Lei ํo 8.080 , de 19 de setembro de 1990 e o mais recente Decreto oㅜ 7.508 , de 2011, reafirmaram estes pressupostos (3) e tornaram legítimo que qualquer cidadão possa impetrar junto ao Poder Judiciário trâmites para conceder benefícios não garantidos pelas políticas públicas de saúde vigentes.

Ampliou-se desde então o fenômeno denominado 'Judicialização da Saúde', referido por alguns autores como o deslocamento do pólo de decisão de certas questões que tradicionalmente eram prerrogativas dos Poderes Legislativo e Executivo para o âmbito do Judiciário (4). As principais demandas têm sido observadas quanto a medicamentos, assistência à saúde e outros insumos (5), embora desde a década de 90 se configuraram reivindicações por medicações e procedimentos médicos entre as pessoas que viviam com HIV/Aids, sendo este citado com o marco histórico do início da judicialização da saúde no Brasil (6).

Em virtude da ampla advocacia empreendida pelas organizações nãogovernamentais, fez-se jurisprudência favorável à responsabilização dos entes federativos no cumprimento imediato desta prestação estatal e em avanços nas políticas públicas de saúde às pessoas com HIV/Aids, principalmente ao acesso universal e gratuito aos medicamentos antirretrovirais (6).

Tais avanços serviram de incentivo ao uso Poder Judiciário como forma de garantia de direitos e até mesmo como forma de ampliação de políticas públicas. Exemplo disto é que no Estado do Rio de Janeiro, em 1988, o HIV era a patologia com predominância nas ações judiciais, com um índice superior a 90,0\%, porém nos anos 2000 reduziu este índice para $14,6 \%(7)$.

Apesar de o Estado manter uma estrutura complexa e aporte financeiro para garantir o cumprimento do seu dever de ofertar ações e serviços de saúde de forma universal e equitativa, nem sempre há garantia de sua efetividade. O cumprimento dos despachos judiciais pelos entes federativos impacta em ampliação dos custos, não previstos em 
orçamento (8), sobretudo quanto aos medicamentos essenciais (9).

Os medicamentos são a intervenção terapêutica mais utilizada e constituem uma tecnologia que exerce alto impacto sobre os gastos em saúde. Por isso, no Sistema Único de Saúde (SUS), a otimização dos processos que levam ao acesso dos medicamentos aos usuários, fica a cargo da área da Assistência Farmacêutica que classifica os medicamentos essenciais à saúde em três componentes: básico, estratégico e especializado (10).

Atualmente, a judicialização na saúde se tornou um dos principais problemas enfrentados pelas gestões estaduais do SUS (10), tendo os medicamentos como maiores objetos das ações judiciais (11).

Considerando a crescente discussão sobre a Judicialização da Saúde, à escassez de publicações sobre o tema no estado de Pernambuco e os desafios que traz para a gestão do Sistema Único de Saúde, o presente artigo objetivou analisar a judicialização do acesso aos serviços de saúde na Secretaria Estadual de Saúde de Pernambuco no ano de 2016.

\section{Métodos}

Tratou-se de estudo transversal, quantitativo e analítico, cuja fonte para a coleta dos dados foi o banco disponibilizado pelo Núcleo de Ações Judiciais (NAJ) da Secretaria Estadual de Saúde de Pernambuco (SES/PE). O recorte temporal estabelecido foi o ano de 2016, em que ocorreram 2.560 processos.

Os dados foram tabulados através do programa Excel for Windows. As variáveis de interesse escolhidas foram: o produto; a unidade de atendimento (se SUS, rede privada e filantrópico); condição patológica segundo a Classificação Estatística Internacional de Doenças e Problemas Relacionados com a Saúde 10ª Revisão (CID-10); municípios da comarca; município de residência; sexo; grupo do produto (se medicamento, UTI, alimento, insumo, material médico-hospitalar, cirurgia, equipamento, homecare, exame, consulta, Órteses, Próteses e Materiais Especiais, internamento e outros) e condutor da ação (se Defensoria Pública da União (DPU), Ministério Público da União (MPU), Associação de Defesa dos Usuários de Seguros, Planos e Sistemas de Saúde (ADUSEPS), Defensoria Pública do Estado de Pernambuco (DPPE), Ministério Público de Pernambuco (MPPE e particular).

Nas análises estatísticas foram calculadas as frequências simples e os percentuais 
de todas as variáveis, identificando as principais características das demandas judiciais. No segundo momento, foi realizado o cruzamento das seguintes variáveis: produto versus condição patológica segundo a CID-10; produto versus a unidade de atendimento; condição patológica segundo a CID-10 versus Região de Saúde de residência; condutor da ação versus produto; condutor da ação versus Região de Saúde de residência e produto versus Região de Saúde de residência.

Para observação do comportamento dos medicamentos, objeto das ações, avaliou-se a listagem de medicamentos, bem como os ditos medicamentos do componente especializado da rotina de aquisição e distribuição do Estado de Pernambuco, pela sua diretoria da Assistência Farmacêutica, sendo compreendidos como medicamentos de alto custo.

\section{Resultados e discussão}

Dentre as demandas judiciais relacionadas à saúde no Estado de Pernambuco, destacam-se os medicamentos, que no ano de 2016 corresponderam a 63,5\% das ações judiciais impetradas contra a SES/PE (tabela 1). Este cenário corrobora com os estudos encontrados na literatura, ao afirmarem que os medicamentos para uso individual são os principais objetos de judicialização na área da saúde (7). Identificou-se que nos últimos anos houve um aumento considerável no número de processos judiciais individuais e coletivos principalmente como via alternativa de aquisição de medicamentos no SUS (12).

Tabela 1. Demandas judiciais por Categoria. Pernambuco, 2016.

\begin{tabular}{lcc}
\hline Categoria & $\mathrm{N}$ & $\%$ \\
\hline Medicamentos & 1626 & 63,5 \\
UTI & 207 & 8,1 \\
Alimento & 131 & 5,1 \\
Insumos & 119 & 4,6 \\
Cirurgia & 94 & 3,6 \\
Material Médico-hospitalar & 86 & 3,4 \\
Equipamento & 73 & 2,8 \\
Homecare & 66 & 2,6 \\
Exames & 55 & 2,1 \\
Consulta & 36 & 1,4 \\
Órteses, Prótese, e Materiais Especiais & 25 & 1 \\
\hline
\end{tabular}




\begin{tabular}{|c|c|c|}
\hline Outros & 23 & 1 \\
\hline Internamento & 18 & 0,7 \\
\hline Não informado & 1 & 0,04 \\
\hline Total & 2560 & 100 \\
\hline
\end{tabular}

Fonte: Banco de dados com as ações judiciais do ano de 2016, do Núcleo de Ações Judiciais - NAJ da Secretaria Estadual de Saúde de Pernambuco- SES/PE. Elaboração própria

Da demanda por medicamentos no ano de 2016 em PE, identificou-se 831 medicamentos distintos ${ }^{11}$, sendo $28 \%$ pertencentes ao Componente Especializado da Assistência Farmacêutica (CEAF) e 13\% correspondem a lista de medicamentos considerados de alto custo para aquisição. Sendo mais restrito, dos medicamentos ditos do grupo do CEAF, 48\% são denominados 'alto custo de aquisição' (tabela 02).

Analisando o quantitativo dos medicamentos adquiridos por ações judiciais, identificou-se 1.560 itens de medicamentos adquiridos. Número maior que o tipo de medicamento (831), pois um tipo de medicamento gera quantidade igual ou maior de unidades/doses adquiridas. Continuando a análise, (tabela 02), 42\% dos medicamentos adquiridos foram pertencentes ao CEAF, sendo 30\% correspondem a lista de medicamentos considerados de alto custo para aquisição do estado. Sendo mais restrito, dos medicamentos ditos do grupo do CEAF, $72 \%$ do quantitativo são denominados 'alto custo de aquisição'.

Tabela 2. Comportamento das ações judiciais pela categoria Medicamentos. Pernambuco, 2016.

\begin{tabular}{|l|c|c|l|c|c|}
\hline $\begin{array}{l}\text { Medicamentos } \\
\text { Itens diferentes }\end{array}$ & & $\begin{array}{l}\text { Medicamentos } \\
\text { Unidades adquiridas }\end{array}$ & & \\
\hline Total & 831 & $100 \%$ & Total & 1560 & $100 \%$ \\
\hline CEAF & 231 & $28 \%$ & CEAF & 658 & $42 \%$ \\
\hline Alto custo & 111 & $13 \%$ & Alto custo & 472 & $30 \%$ \\
\hline & & & & & \\
\hline CEAF & 231 & $100 \%$ & CEAF & 658 & $100 \%$ \\
\hline Alto custo (segundo & 111 & $48 \%$ & Alto custo (segundo DGAF- & 472 & $72 \%$ \\
\hline
\end{tabular}

Fonte: Banco de dados com as ações judiciais do ano de 2016, do Núcleo de Ações Judiciais - NAJ da Secretaria Estadual de Saúde de Pernambuco- SES/PE. Elaboração própria

Verticalizando a análise, os 5 medicamentos mais requisitados foram: Cinacalcete,

\footnotetext{
${ }^{11}$ Para fins de maior compreensão dos leitores, considerou-se neste, fármacos distintos o mesmo de medicamentos distintos, seja por fórmula (princípios ativos distintos ou concentrações diferentes do mesmo princípio ativo), seja por forma/apresentação (distinções entre cápsulas, comprimidos, gel, pomada, creme entre outras).
} 
Somatropina, Abiraterona, Insulina Glargina e Leuprorrelina. O Cinacalcete (Mimpara®) é o item medicamentoso mais judicializado no Estado de Pernambuco, correspondendo a $4,5 \%$ das demandas. O fármaco é indicado para o tratamento de pacientes com Hiperparatireoidismo secundário (HPTS) à doença crônica renal (DRC), que são submetidos à diálise com paratormônio (PTH) superior a 800 pg/mL. Este foi incorporado através da Portaria № 48 da Secretaria de Ciência, Tecnologia e Insumos Estratégicos do Ministério da Saúde em 29 de setembro de 2015, com prazo de 180 dias para incorporação. Contudo, em novembro de 2016 o Protocolo Clínico e Diretrizes Terapêuticas Específico foi aberto para uma segunda consulta pública (13), sendo incorporado no final de 2017, iniciando sua distribuição no primeiro trimestre de 2018, podendo explicar o crescente número de demandas judiciais para aquisição do deste.

Em segundo lugar está a Somatropina (3,4\%) que se trata de um hormônio estimulador e acelerador do crescimento em crianças com deficiência de hormônio do crescimento endógeno. A droga está disponível no SUS através do CEAF para o tratamento de Hipopituitarismo e Síndrome de Turner (14).

Seguida da Somatropina está a Abiraterona (Zytiga®), equivalente a 3,4\% das demandas de medicamentos, utilizado no tratamento do câncer de próstata em razão de diminuir a produção dos androgênios (hormônios sexuais), assim retardando a progressão da doença, devendo ser administrado concomitantemente com a droga Prednisona (ou Prednisolona) a fim de prevenir o Hiperparatireoidismo secundário (HPTS), que se caracteriza como um dos efeitos colaterais do medicamento. É importante frisar que a Tabela SUS não refere medicamentos oncológicos, mas situações tumorais particulares, a fim de orientar a definição de determinado tratamento, sendo de responsabilidade dos hospitais habilitados em Oncologia e credenciados no SUS padronizar, adquirir e fornecer tais medicamentos. O fármaco ainda não compõe a Relação Nacional de Medicamentos Essenciais (RENAME), no entanto o tratamento oncológico é assegurado em sua totalidade pelo SUS, sendo assim, as unidades de saúde credenciadas em Oncologia devem ser ressarcidas por suas respectivas Secretarias de Saúde. (13)

A Insulina Glargina, registrada na Agência Nacional de Vigilância Sanitária (Anvisa) para uso no tratamento do diabetes mellitus tipo 2 em adultos e tratamento de diabetes mellitus tipo 1 em adultos e crianças com dois anos ou mais que precisam de insulina basal para controle da hiperglicemia, está em quarto lugar (2,9\%) entre os cinco 
medicamentos mais judicializados no Estado. Deve ser levado em consideração que no período de análise foi observado o aumento das ações entre os meses de maio a setembro, que correspondem aos meses em que a insulina glargina estava em falta na Farmácia do Estado. A substância não está disponível nacionalmente através do SUS, porém no Estado de Pernambuco foi incorporada ao serviço por meio de uma Ação Civil Pública, em resposta ao crescente número de demandas judiciais para aquisição da mesma. (15)

Em quinto lugar está a Leuprorrelina, representando 2,6\% dos casos. De acordo com o Ministério da saúde, a droga é:
Um nonapeptídeo sintético análogo do hormônio liberador da gonadotrofina natural ( $\mathrm{GnRH}$ ou LH-RH). Possui maior potência que o hormônio natural, atua como um inibidor da produção de secreção de gonadotrofina, quando administrado continuamente e em doses terapêuticas e é quimicamente distinto dos esteroides. (16)

O medicamento está inserido na lista de Assistência Farmacêutica do SUS para o tratamento de Leiomioma de Útero, Puberdade Precoce Central e Endometriose através do CEAF (15).Para entender esse processo é importante ressaltar que segundo o CONASS (2011), o setor saúde está correlacionado com os avanços científicos e tecnológicos, portanto precisa-se levar em consideração o interesse da indústria farmacêutica, que utilizando de estratégias de marketing lançam rotineiramente novos medicamentos com pequenas alterações em suas fórmulas, a fim de incentivar a classe médica e aos usuários a trocarem os medicamentos já disponíveis por medicamentos novos que prometem melhores benefícios.

Quando distribuídas as demandas nas 12 regiões de saúde, observa-se que $42 \%$ concentraram-se na $1^{\text {a }}$ Região de Saúde (figura 1), que abriga a Região Metropolitana do Recife. Esse fato pode ser justificado pelo grande aglomerado de pessoas na região como também pela concentração de unidades de saúde de média e alta complexidade. Além disso, foi observado que as unidades de origem das ações em sua maioria do Sistema Único de Saúde e Filantrópicos, representando 39,3\% e 31,5\% respectivamente. A rede privada representa 19,3\%, conforme gráfico 1. 
Figura 1. Demandas judiciais por Regiões de Saúde do estado de Pernambuco, 2016.

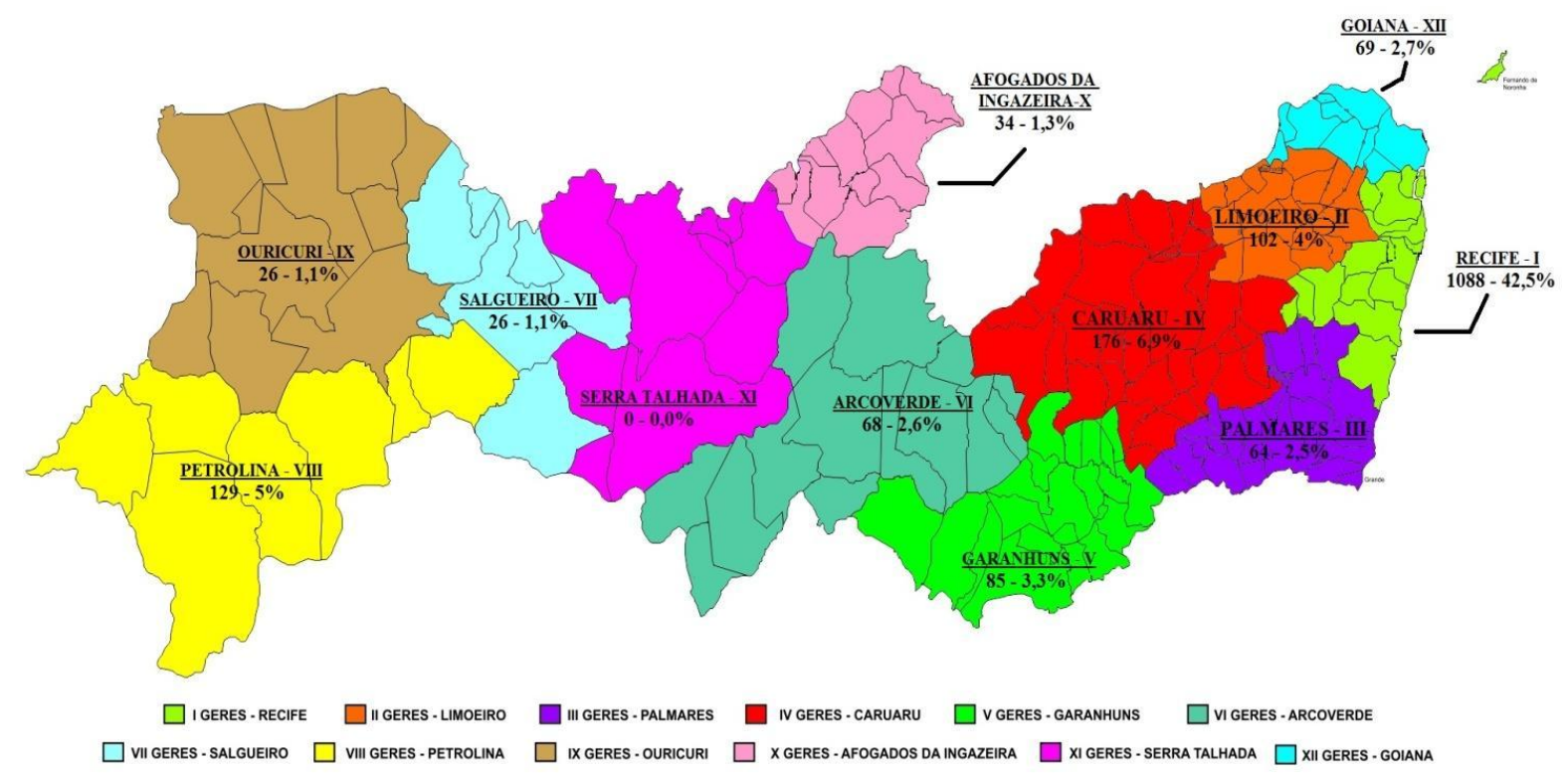

Fonte: Banco de dados com as ações judiciais do ano de 2016, do Núcleo de Ações Judiciais - NAJ da Secretaria Estadual de Saúde de Pernambuco- SES/PE.

Nota: $27 \%$ das ações judiciais no banco não tinham a variável preenchida.

Gráfico 1. Ações judiciais por unidade de saúde demandante. Pernambuco, 2016.

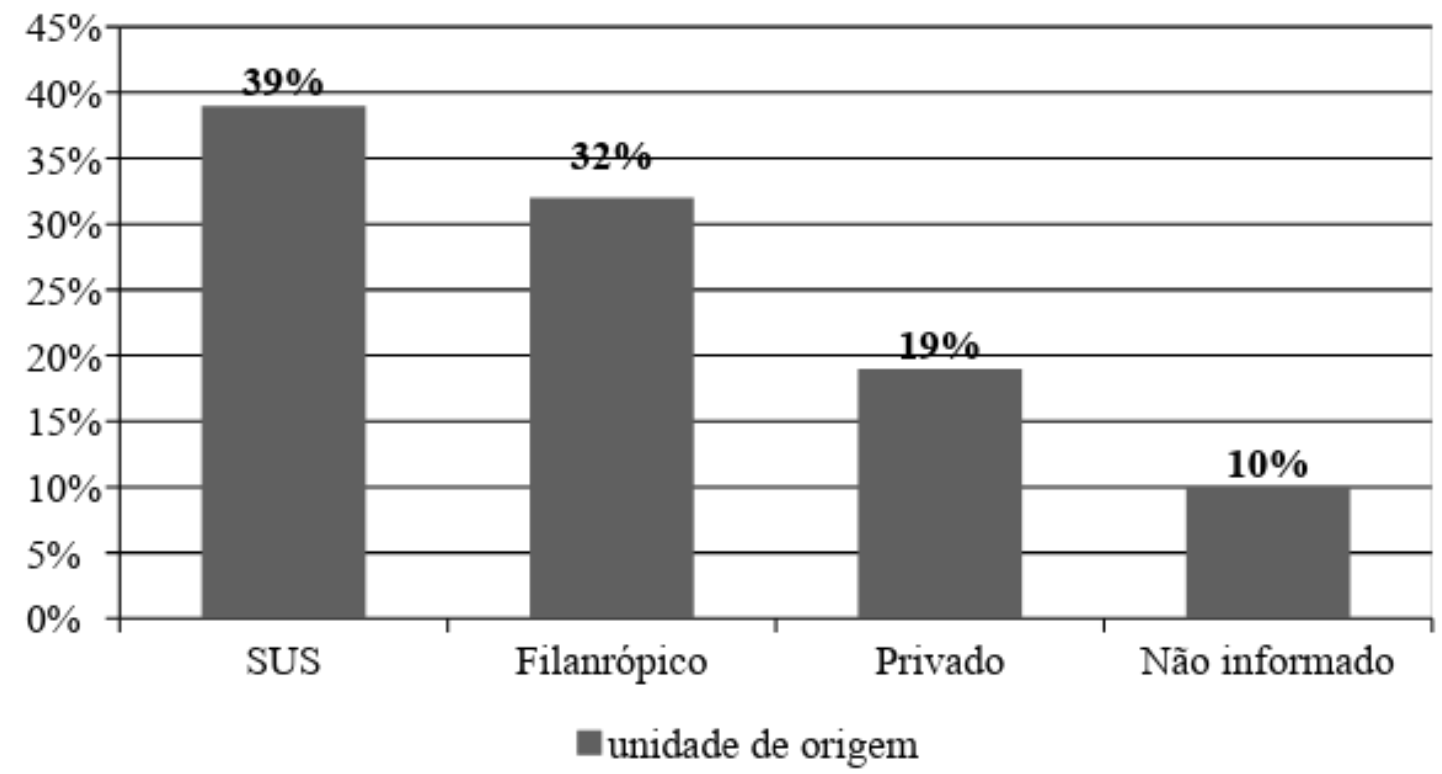

Fonte: Banco de dados com as ações judiciais do ano de 2016, do Núcleo de Ações Judiciais - NAJ da Secretaria Estadual de Saúde de Pernambuco- SES/PE

Quando analisado o número de demandas por mês no ano de 2016 (gráfico 2), observou-se que o número de ações judiciais mantém padrão em sua distribuição, com 
aumento entre os meses de julho a novembro, porém não foram encontrados na literatura argumentos relevantes que possam explicar esse evento.

Gráfico 2. Ações judiciais por mês de ocorrência. Pernambuco, 2016.

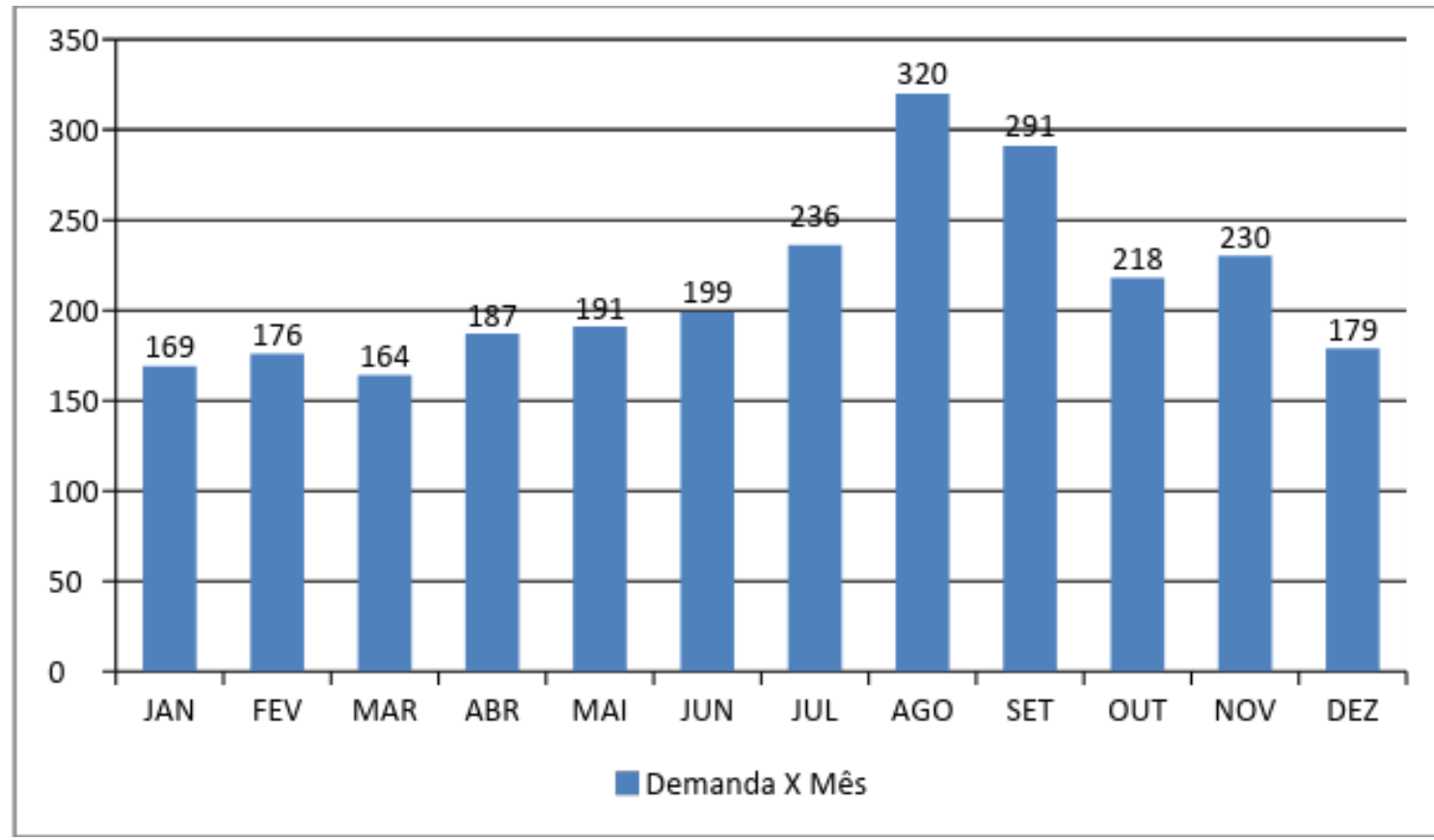

Fonte: Banco de dados com as ações judiciais do ano de 2016, do Núcleo de Ações Judiciais - NAJ da Secretaria Estadual de Saúde de Pernambuco- SES/PE

No Estado, as vias de acesso à Judicialização da Saúde se dão comumente através da Defensoria Pública do Estado de Pernambuco (DPPE); Ministério Público de Pernambuco (MPPE); Defensoria Pública da União (DPU); Associação de Defesa dos Usuários de Seguros, Planos e Sistemas de Saúde e Advogados Particulares. Diferentemente de estudos que apontam a Judicialização como um processo das elites brasileiras (17), no Estado de Pernambuco durante o período de análise foi observado que as demandas judiciais em sua maioria foram patrocinadas por Órgãos Públicos, correspondendo a $54,6 \%$ dos casos, sendo a DPPE o órgão com maior representatividade no número de casos, com 36,8\%. Ainda assim, observou-se que os Advogados Particulares representam 29,3\%. A partir desta análise é possível identificar que a realidade pernambucana assemelha-se com a encontrada por Diniz, em seu estudo realizado no Distrito Federal afirmando que entre os anos de 2005 a 2010, 95\% dos processos foram encaminhados por defensores públicos. (5) 
Gráfico 3. Ações judiciais por órgão patrocinador. Pernambuco, 2016.

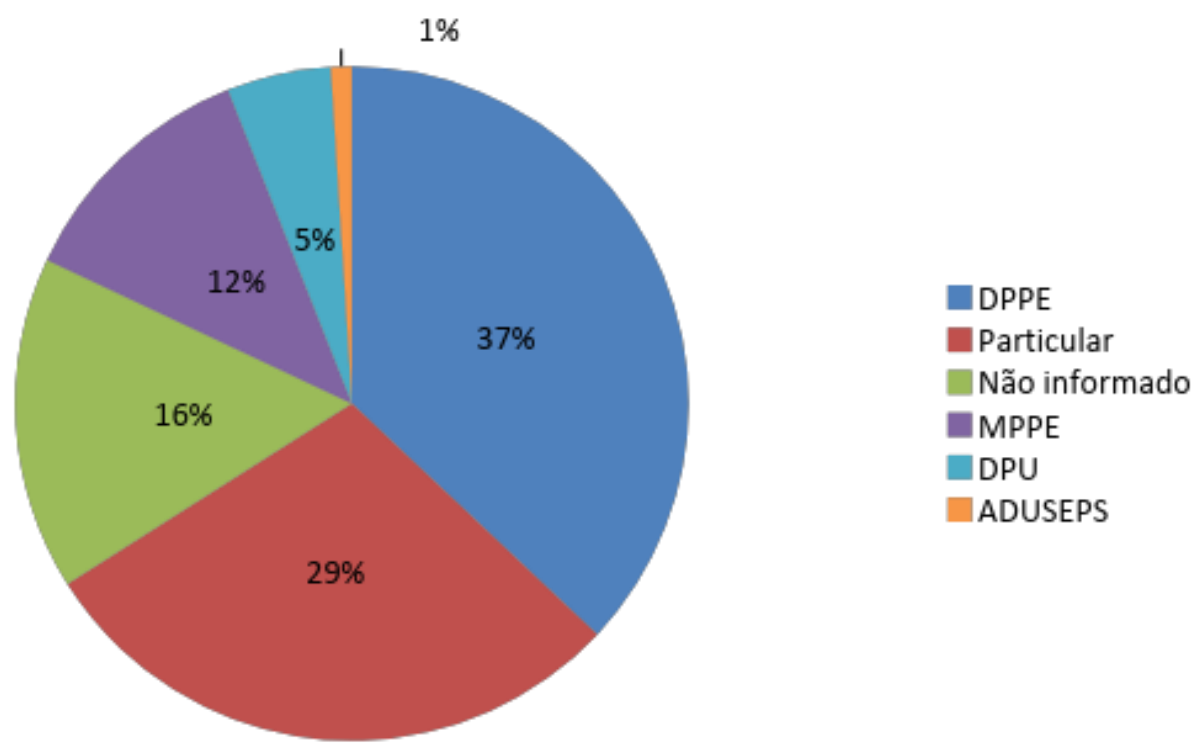

Fonte: Banco de dados com as ações judiciais do ano de 2016, do Núcleo de Ações Judiciais - NAJ da Secretaria Estadual de Saúde de Pernambuco- SES/PE

Das 2.560 ações judiciais impetradas contra a Secretaria de Saúde de Pernambuco no ano de 2016, 2.438 foram deferidas, correspondendo a 95,2\% e apenas 4,5\% (115) tiveram o pedido negado. O grande número de ações deferidas no Estado chama a atenção para a formação do magistrado, no que diz respeito à análise de processos na área da saúde, levando em consideração que o tema requer conhecimentos específicos. Além disso, é importante ressaltar o estudo de Marcos Maselli (18) que acrescenta que o Poder Judiciário pode não levar em consideração algumas implicações relevantes da sentença, pois não analisa os processos em sua total complexidade.

Em função das limitações do Poder Judiciário frente ao tema (10) e do número crescente de demandas judiciais relacionadas à saúde, o Conselho Nacional de Justiça (CNJ) através da Recomendação № 31 de 31 de março de 2010, propôs a implementação de medidas para subsídio de magistrados e demais operadores do direito a fim de garantir melhor eficácia na solução das demandas judiciais que envolvem a saúde.

Em resposta à recomendação do $\mathrm{CNJ}$, o estado de Pernambuco implementou o Núcleo de Assistência Técnica em Saúde (NATS), sendo resultado de uma parceria entre Tribunal de Justiça de Pernambuco (TJPE) e o Poder Executivo. O NATS/PE é atualmente 
composto por uma equipe técnica formada por uma advogada, uma médica e uma farmacêutica, funcionando como uma ferramenta de apoio aos magistrados, para permitir maior esclarecimento a respeito das demandas judiciais da saúde (19).

\section{Considerações Finais}

A Judicialização da Saúde no Estado de Pernambuco é caracterizada pelo crescente número de demandas judiciais por medicamentos, configurando-se como via de acesso alternativa para aquisição destes no SUS, uma vez que os fármacos mais requisitados estão incorporados na lista de Assistência Farmacêutica do Ministério da Saúde.

Houve concentração das demandas judiciais do Estado na $1^{\text {a }}$ Região de Saúde, vindas predominantemente de unidades de saúde do SUS e patrocinadas comumente por órgãos públicos, não corroborando com teses que afirmam que a judicialização se caracteriza como um movimento da elite brasileira. Além disso, o estudo reafirma a necessidade do incentivo e investimentos em pesquisas relacionadas ao tema, tendo em vista a complexidade e relevância do assunto para a saúde pública brasileira.

\section{Referências}

1. Oliveira LM, Andrade EIG, Milagres MO. Ministério Público e políticas de saúde: implicações de sua atuação resolutiva e demandista. Revista de Direito Sanitário. 2015, 15(3):142-161. Disponível em: http://www.revistas.usp.br/rdisan/article/view/97332 Acesso em 29.5.2018.

2. Brasil. Constituição da República Federativa do Brasil de 5 de outubro de 1999. Brasíia: Senado Federal, 2013.

3. Brasil. Lei no 8.080, de 19 de setembro de 1990. Dispõe sobre as condições para a promoção, proteção e recuperação da saúde, a organização e o funcionamento dos serviços correspondentes e dá outras providências. Disponível em: http://www.planalto.gov.br/ccivil 03/leis/L8080.htm Acesso em 25.4.2018.

4. Tate CN, Vallinder T (orgs). The global expansion of judicial power. New York: NYU Press, 1997.

5. Diniz D, Machado TRDC, Penalva J. A judicialização da saúde no Distrito Federal, Brasil. Ciência \& Saúde Coletiva. 2014,19(2): 591-598. Disponível em:

http://www.scielo.br/scielo.php?pid=S141381232014000200591\&script=sci abstract\&tlng= pt Acesso em 29.5.2018.

6. Ventura M, Simas L, Pepe VLE, Schramm FR. Judicialização da saúde, acesso à 
justiça e a efetividade do direito à saúde. Physis: Revista de Saúde Coletiva. 2010, 20(1):77-100. Disponível em: http://www.scielo.br/scielo.php?script=sci arttext\&pid=S010373312010000100006 Acesso em 29.5.2018.

7. Messeder AM, Osorio-de-Castro CGS, Luiza VL. Mandados judiciais como ferramenta para garantia do acesso a medicamentos no setor público: a experiência do Estado do Rio de Janeiro, Brasil. Cadernos de Saúde Pública. 2005, 21(2):525-534. Disponível em: http://www.scielo.br/scielo.php?script=sci arttext\&pid=S0102311X2005000200019 Acesso em 29.5.2018.

8. Massaú GC, Bainy AK. O impacto da judicialização da saúde na comarca de Pelotas. Revista de Direito Sanitário. 2015, 15(2):46-65. Disponível em: http://www.revistas.usp.br/rdisan/article/view/88357 Acesso em 29.5.2018.

9. Vieira FS, Zuchi P. Distorções causadas pelas ações judiciais à política de medicamentos no Brasil. Revista de Saúde Pública. 2007, 41(2):214-222. Disponível em: http://www.scielo.br/scielo.php?pid=S003489102007000200007\&script=sci abstract\&tlng= pt Acesso em 29.5.2018.

10. Conselho Nacional de Secretários de Saúde. A Assistência Farmacêutica no SUS. Brasília: CONASS, 2011. Disponível em: http://www.conass.org.br/biblioteca/pdf/atualizacao-2015/L07 Assis-Farmaceutica-noSUS jun2015.pdf Acesso em 25.4.2018.

11. Pernambuco. Secretaria Estadual de Saúde. Portal eletrônico da Secretaria Estadual de Saúde. Disponível em: http://portal.saude.pe.gov.br/secretaria Acesso em 25.4.2018.

12. Pepe VLE, Figueiredo TA, Simas L, Osorio-de-Castro CGS, Ventura M. A judicialização da saúde e os novos desafios da gestão da assistência farmacêutica. Ciência \& Saúde Coletiva. 2010, 15(5):2405-2414 . Disponível em: http://www.scielo.br/scielo.php?pid=S1413$81232010000500015 \&$ script $=$ sci abstract\&tIng=pt Acesso em 25.4.2018.

13. Universidade Federal de Minas Gerais. Hospital das Clínicas. Núcleo de Avaliação de Tecnologias em Saúde. Informações Técnicas sobre o Cinacalcete (Mimparaß) para pacientes em tratamento dialítico.2012, 5. Disponível em:

https://bd.tjmg.jus.br/jspui/bitstream/tjmg/4997/1/NATS NT 2012 15\%20Cinacalcete\%20n a\%20Insuficiência\%20renal\%20crônica\%20dial\%C3\%ADtica.pdf Acesso em 25.4.2018.

14. Ministério da Saúde. Consultoria Jurídica/ Advocacia Geral da União. Nota Técnica № 335/2014. Brasília, 2014.

15. Ministério da Saúde. Consultoria Jurídica/ Advocacia Geral da União. Nota Técnica № 26/2012. Brasília, 2012.

16. Ministério da Saúde. Consultoria Jurídica/ Advocacia Geral da União. Nota Técnica 
№ 482/2014MINISTÉRIO DA SAÚDE. Brasília, 2014.

17. Medeiros M, Debora D, Ida VDS. A tese da judicialização da saúde pelas elites: os medicamentos para mucopolissacaridose. Disponível em: http://www.scielo.br/scielo.php?script=sci arttext\&pid=S1413-81232013000400022 Acesso em 25.4.2018.

18. Barroso LR. Da falta de efetividade à judicialização excessiva: direito à saúde, fornecimento gratuito de medicamentos e parâmetros para a atuação judicial. Disponível em: https://www.conjur.com.br/dl/estudobarroso.pdf Acesso em 25.4.2018.

19. Poder Judiciário de Pernambuco. Parceria entre o TJPE e o Poder Executivo auxilia magistrados em ações judiciais relacionadas à saúde. Assessoria de Comunicação do Tribunal de Justiça de Pernambuco. Disponível em:

https://www.tjpe.jus.br/intranet/noticias ascomSY/ver noticia.asp?id=9762 Acesso em: 25.4.2018.

20. Figueiredo TA, Schramm JMA, Pepe VLE. Seleção de medicamentos essenciais e a carga de doença no Brasil. Cadernos de Saúde Pública. 2014, 30(11):2344-2356. Disponível em: http://www.scielo.br/scielo.php?pid=S0102311X2014001102344\&script=sci abstract\&tlng= pt Acesso em 25.4.2018.

Como citar este artigo:

Oliveira FHC, Lorena Sobrinho JE, Lima MCS, Montarroyos UR, Neves MGAB, Silva PR, Xavier LN, Jorge Filho G, Guedes DM, Almeida FJC. Judicialização do Acesso aos Serviços de Saúde: análise de caso da Secretaria de Saúde de Pernambuco. Revista Cadernos Ibero-Americanos de Direito Sanitário. 2018 abr./jun, 7(2):173-186. 